\title{
Reeducación psicomotriz en personas con enfermedad de Alzheimer
}

\author{
BERNAT-CARLES SERDÀ I FERRER ${ }^{1, a}$, \\ ASSUMPTA ORTIZ COLLADO ${ }^{1, b}$, PILAR ÀVILA-CASTELLS ${ }^{1,2}$
}

\section{Impact of a psychomotor re-education guide on the quality of life of patients with Alzheimer's disease}

Background: We developed a psychomotor re-education guide (PSEG) adapted to people with Alzheimer disease $(A D)$, including a cognitive stimulation program integrated with the exercise recommendations of the American College of Sports Medicine. Aim: To evaluate the effect of the PSEG on cognitive and functional parameters among patients with AD. Patients and Methods: We applied the PSEG to 64 participants with AD aged 64 to 87 years (55\% women) during 12 months. At baseline and the end of the intervention, cognitive abilities (Mini Mental State), fitness (Chair Stand test), level of independence (Barthel Index) and quality of life (QoL) (SF-12) were assessed. Results: The application of PSEG resulted in a significant improvement in the QoL and physical capacity of patients with AD. Cognitive ability declined along with disease progression. Conclusions: These results demonstrate the effectiveness of the guide in mild and moderate stages of $A D$ improving the physical fitness and the QoL.

(Rev Med Chile 2013; 141: 735-742).

Key words: Alzheimer Disease; Exercise; Physical fitness; Psychomotor disorders.

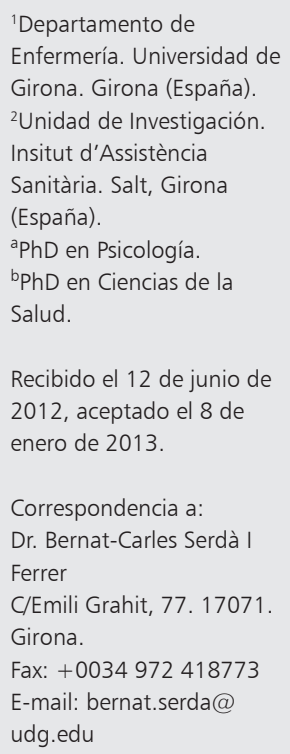

E n el mundo, aproximadamente 35 millones de personas sufren demencia. Se calcula que en el 2030, 66 millones de personas podrían padecer algún trastorno neurodegenerativo ${ }^{1}$. España se encuentra entre los cinco países europeos que presenta más casos de enfermedad de Alzheimer (EA) con una prevalencia de 1,36\%, ligeramente superior a la media europea, de $1,27 \%{ }^{2}$.

La EA se caracteriza por una pérdida insidiosa de la facultad cognitiva, de las alteraciones conductuales anímicas y psiquiátricas que generan un estado de dependencia ${ }^{3}$. Entre las facultades cognitivas afectadas destacamos la pérdida temprana de la capacidad de fijar nueva información y recordar acontecimientos (amnesia); reconocer los objetos en su entorno (agnosia); comunicarse, expresarse y entender (afasia). Un factor que incide en la dependencia de la persona mayor con EA es la pérdida progresiva de la capacidad física y funcional, impidiendo la realización de las actividades de la vida cotidiana (apraxia) ${ }^{4}$. Este estado impacta en la calidad de vida (CdV) de los enfermos y en la de sus cuidadores ${ }^{5-7}$.

La eficacia del tratamiento farmacológico es limitada y no consigue detener el deterioro cognitivo asociado a las demencias ${ }^{8,9}$. Por este motivo, ha aumentado el interés hacia el diseño y experimentación a partir de nuevas modalidades de tratamiento no farmacológicas. Las terapias complementarias corresponden a técnicas de intervención con una aproximación holística e integradora que tienen como objetivo mejorar el bienestar general de las personas enfermas ${ }^{10,11}$. El ejercicio físico es un tipo de terapia complementaria en que la práctica habitual tiene un efecto beneficioso para la salud en la $\mathrm{EA}^{12}$. Sus efectos se circunscriben a nivel físico y funcional ${ }^{13,14}$ mejorando el mantenimiento de la autonomía, 
la disminución de la carga de los cuidadores y el riesgo de institucionalización ${ }^{15}$.

García-Mesa (2011) confirma que el ejercicio físico tiene un efecto neuroprotector sobre la actividad de la corteza cerebral, lo que se traduce en un aumento de la memoria a largo plazo y en una mayor capacidad de aprendizaje complejo ${ }^{16}$.

Penrose (2005) determina que la característica más importante de la práctica de ejercicio con pacientes con EA es la intencionalidad, minimizando el automatismo y la repetición ${ }^{17}$. Esta premisa mejora el síndrome afaso-apraxo-agnósico asociado a la enfermedad. En esta línea, una estrategia de intervención en experimentación consiste en integrar el ejercicio físico con la estimulación cognitiva en un marco social saludable ${ }^{17,18}$. Sin embargo, deben diseñarse guías adaptadas a la EA que concreten los factores que determinan la dosis de actividad física más eficaz ${ }^{19}$.

En base a lo expuesto, este estudio plantea dos objetivos principales:

a) Diseñar e implementar una guía de reeducación psicomotriz (GREPS) adaptada a las fases leve, moderada y grave de las personas con EA.

b) Evaluar los efectos de la GREPS a nivel físico, funcional, cognitivo y en la CdV de los participantes del estudio.

\section{Pacientes y Métodos}

Se realizó un estudio utilizando un diseño casi experimental, con medición de las variables antes y después de un período de observación. La muestra se compuso de 64 participantes con EA seleccionados aleatoriamente a partir de la historia clínica y aplicando el programa SPSS v.15.

\section{Procedimientos}

Después de obtener el informe favorable por parte del comité ético de investigación clínica (CEIC) de referencia, el programa de ejercicio se puso en marcha en los hospitales de día del Alt y Baix Empordà desde el mes de junio de 2008 hasta el mes de octubre de 2009.

Se incluyeron en el programa los pacientes con EA que cumplían los siguientes criterios de inclusión:

1. Diagnóstico neuropsicológico de EA en cualquier fase de la patología confirmado por el facultativo médico a partir de los resultados del $\mathrm{MMS}^{20} \mathrm{y}$ en acorde a los criterios de diagnóstico internacionales estandarizados ${ }^{21}$.

2. Superación del examen médico preintervención en el que se declaraba que el enfermo no presentaba ninguna de las contraindicaciones incompatibles con el programa de ejercicio.

3. Obtención del consentimiento informado del interesado/a y/o cuidador/a.

Los criterios de exclusión fueron:

1. Presentar alguna de las enfermedades mentales siguientes: esquizofrenia, depresión, trastorno obsesivo-compulsivo (TOC).

2. Modificar la prescripción de tratamiento durante la duración del estudio.

3. La presencia de cualquier patología que contraindique la práctica temporal o permanente de ejercicio físico, destacando como contraindicaciones absolutas la existencia de insuficiencia cardiaca en fase III o IV según la clasificación de la New York Heart Association (NYHA), la hipertensión arterial descompensada y el dolor agudo.

4. Drogodependencias.

5. La imposibilidad de entender o hablar el español.

\section{Diseño de la GREPS}

La finalidad de la GREPS es rehabilitar las dimensiones motriz, cognitiva, afectiva y social. Por este motivo su diseño es modular, e integra tres modalidades terapéuticas: a) ejercicio físico; b) reeducación cognitiva; c) estimulación psicomotriz. Este diseño modular permite adaptar la guía a las fases de la enfermedad y calibrar las actividades al estado comórbido de los enfermos.

Los objetivos de cada modalidad de intervención son:

a) Mejorar el condicionamiento físico en base a las recomendaciones de la American College Sports Medicine-Position Stand ${ }^{22}$ destinadas a las personas mayores sanas, en las que se combina el ejercicio aeróbico con el de fuerza en un marco psicosocial favorable.

b) Reeducar la capacidad cognitiva. Las actividades de psicoestimulación cognitiva se sustentan en la propuesta de Tárraga y Boada, considerando seis líneas básicas de acutación: la orientación espacial y temporal, el lenguaje y el simbolismo, las praxias, las gnosias, la memoria y el cálculo ${ }^{23}$. La 
GREPS mantiene el diseño de las actividades en un contexto psicomotriz vivencial. Este diseño final introduce el componente motor, lúdico, social y motivacional como valor añadido.

c) Favorecer la estimulación psicomotriz. La estructura de la sesión sigue las directrices de la guía de reeducación psicomotriz para las personas mayores afectadas con $\mathrm{EA}^{24}$.

El diseño de las actividades psicomotrices integra los módulos a) y b) descritos anteriormente. Se priorizan tres conductas psicomotrices fundamentales:

- Las conductas motrices de base (actividades tónico-posturales equilibradas, incluyendo la coordinación dinámica general y visomotora);

- Las conductas perceptivo-motrices (orientación espacial y estructuración temporal);

- Las conductas neuromotrices (lateralidad, sincinesias y paratonías).

La duración del programa de intervención fue de 12 meses en acorde con otros estudios simila$\mathrm{res}^{25}$. Se llevaron a cabo tres grupos independientes considerando las tres fases de la enfermedad: leve $(n=19)$, moderada $(n=20)$ y grave $(n=25)$. La frecuencia de trabajo fue de dos sesiones semanales. Las variables que determinaron la dosis de ejercicio corresponden a una intensidad moderada (70-75\% de la frecuencia cardiaca máxima); la duración de la sesión fue de $60 \mathrm{~min}$, distribuidos en: 10-15 min (fase inicial), 30-35 min (fase de desarrollo), 10-15 min (fase final-feedback).

\section{Evaluación de variables directas}

A partir de la anamnesis clínica se identificó cualquier contraindicación al ejercicio. En el pretest se aplicó un cuestionario sociodemográfico y la evaluación de las variables dependientes que corresponden a: la valoración cognitiva, la condición física, la valoración de la autonomía en las actividades básicas de la vida diaria (ABVD) y la CdV utilizando los siguientes instrumentos:

- Cuestionario sociodemográfico para registrar las variables edad, sexo, estado civil, situación laboral y estadio de la enfermedad.

- Mini Mental State Examination (MMSE) en versión en español [Rango: 0-30] para la valoración cognitiva-conductual ${ }^{26}$.

- Chair Stand Test (Prueba de levantarse de la silla) para determinar la condición física del tren inferior ${ }^{27}$.
- Índice de Barthel para valorar el grado de autonomía ${ }^{28}$.

- Test SF-12 para el estudio de la calidad de vida relativa a la salud (CVRS). Éste valora 8 dimensiones o subescalas de la CVRS: función física, rol físico, dolor corporal, salud general, vitalidad, función social, rol emocional y salud mental ${ }^{29}$.

La aplicación de los cuestionarios Mini Mental State, Chair Stand Test, el índice de Barthel fue realizada por profesionales entrenados específicamente para el estudio. El test SF-12 fue realizado por el cuidador y comprobada por el investigador. Al finalizar el programa de intervención (postest), se evaluaron las mismas variables.

\section{Análisis estadístico}

Para los datos apareados en las variables continuas que seguían una distribución normal, el test estadístico utilizado fue la $t$ de Student-Fisher con un número de grados de libertad de (n-1). Para las variables continuas que no seguían una distribución normal se utilizó el test no paramétrico para datos apareados de Wicolxon o bien, la U de Man Whitney según el tipo de datos. Para analizar las variables categóricas se utilizó el test del $\chi^{2}$. Los análisis estadísticos se realizaron con el programa SPSS versión 15. El nivel de significación escogido fue de $5 \%$.

\section{Resultados}

A partir de los 81 sujetos con EA referidos por el servicio de neurología, se construyó una muestra de 68 participantes del estudio. Durante la intervención cuatro participantes abandonaron el programa. El diagrama de flujo CONSORT representa el proceso de la muestra del estudio (Figura 1).

\section{Características clínicas y epidemiológicas de los participantes}

La edad media del grupo era de 75,53 años con una $\mathrm{DE}=6,28$; rango $=64-87$ ). Del total de participantes, se incluyeron 29 hombres $(45,32 \%)$ y 35 mujeres (54,68\%). La media de edad de las mujeres fue de 77 años $(5,88)$ y la de los hombres 73,55 $(6,08)$. Más de la mitad de participantes estaban casados (67,19\%), 26,56\% eran viudos y $6,25 \%$ 


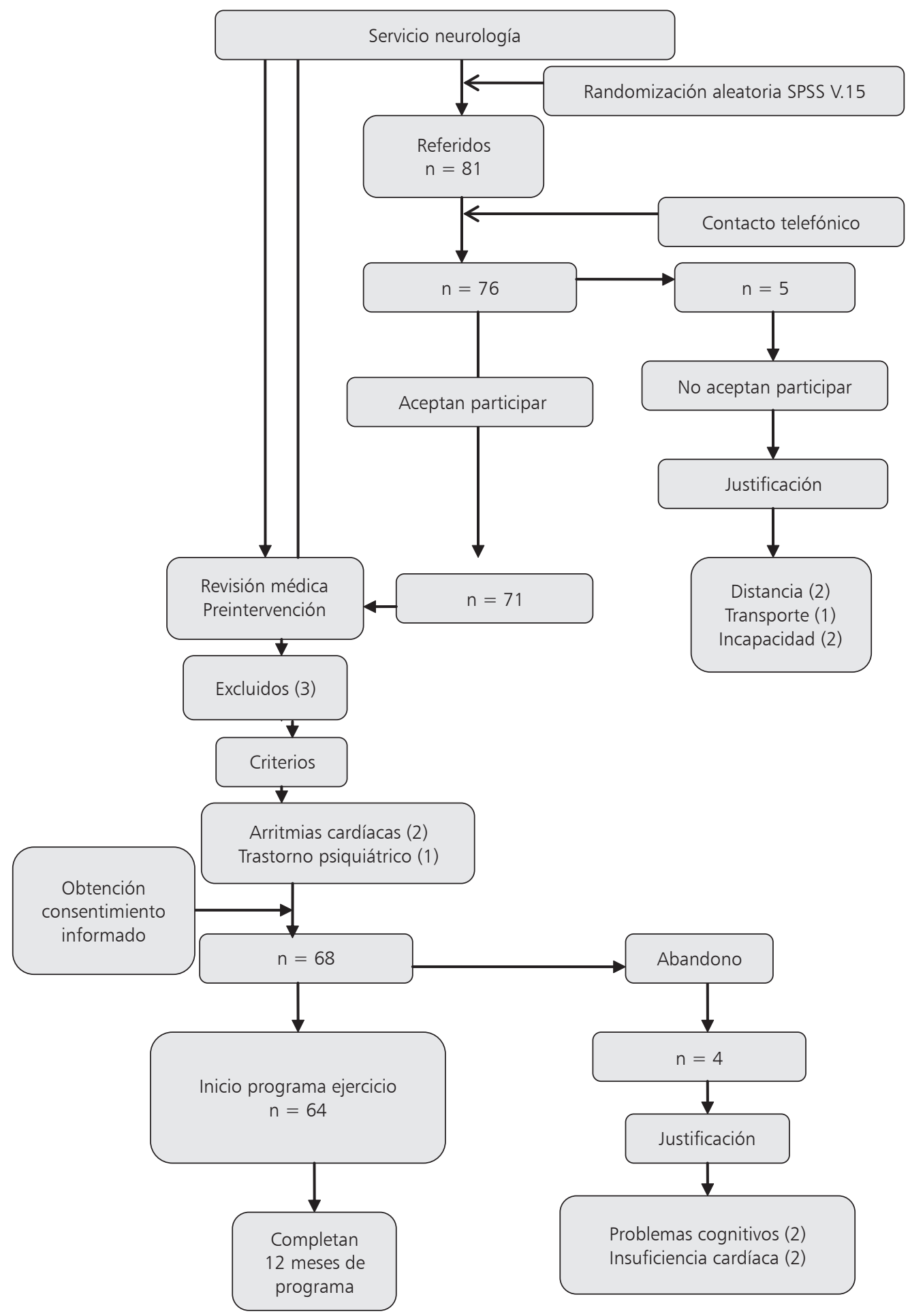

Figura 1. Diagrama de flujo. 
solteros. Con relación a la escolaridad 54,68\% estudió 6 años o más, 34,37\% estudió 5 años o menos y $10,94 \%$ eran analfabetos. Noventa coma sesenta y dos por ciento de los participantes estaban jubilados. Considerando las fases de gravedad de la EA, 19 de los participantes se encontraban en una fase leve, 20 en fase moderada y 25 en fase grave (Tabla 1). La asistencia a las sesiones fue de $73 \%$ (con una media de 58,4 sobre 80 sesiones).

La Tabla 2 muestra el resultado global de los efectos del programa sobre la capacidad física, la capacidad funcional, la capacidad cognitiva y la $\mathrm{CdV}$. El resultado indica que los participantes mejoraron la capacidad física, la capacidad funcional y la CdV. Mientras que la mejora de la capacidad física $(\mathrm{p}=0,012)$ y la $\mathrm{CdV}(\mathrm{p} \leq 0,001)$ fueron significativas, la mejora de la capacidad funcional no lo fue $(\mathrm{p}=0,901)$. Los resultados muestran una disminución significativa de la capacidad cognitiva $(\mathrm{p}=\leq 0,001)$.

En la Tabla 3, se presentan los resultados en relación a cada fase de la enfermedad. Los resultados del test $\mathrm{CdV}$ indican una mejora significativa en las fases leve y moderada de la enfermedad $(\mathrm{p}=\leq 0,001 ; \mathrm{p}=0,008$ respectivamente), mientras que, en la fase grave, la $\mathrm{CdV}$ disminuye de forma no significativa $(p=0,280)$. La capacidad física mejoró en la fase leve de forma significativa ( $\mathrm{p}=\leq 0,001)$; en la fase moderada, también se observó una mejoría, si bien no resultó significativa $(\mathrm{p}=0,664)$. La capacidad cognitiva disminuyó progresivamente en cada una de las fases evaluadas; esta disminución resulta no significativa en la fase leve de la enfermedad ( $\mathrm{p}=0,772)$ y significativa en la fase moderada y grave $(\mathrm{p}=0,022$; $\mathrm{p}=\leq 0,001$ respectivamente). En la variable de la capacidad funcional y autonomía registrada con
Tabla 1. Características clínicas y sociodemográficas de los participantes

\begin{tabular}{|c|c|c|}
\hline Edad, años (DE) & 75,53 & $(6,28)$ \\
\hline \multicolumn{3}{|l|}{ Edad, n (\%) } \\
\hline $60-65$ años & 4 & $(6,25)$ \\
\hline $66-70$ años & 16 & $(25)$ \\
\hline 71 - 75 años & 12 & $(18,75)$ \\
\hline 76 - 80 años & 17 & $(26,56)$ \\
\hline $81-85$ años & 12 & $(18,75)$ \\
\hline+85 años & 3 & $(4,69)$ \\
\hline \multicolumn{3}{|l|}{ Sexo, n (\%) } \\
\hline Hombre & 29 & $(45,31)$ \\
\hline Mujer & 35 & $(54,69)$ \\
\hline \multicolumn{3}{|l|}{ Estado civil, n (\%) } \\
\hline Soltero & 4 & $(6,25)$ \\
\hline Casado & 43 & $(67,19)$ \\
\hline Viudo & 17 & $(26,56)$ \\
\hline \multicolumn{3}{|l|}{ Situación laboral, n (\%) } \\
\hline Activos & 2 & $(3,12)$ \\
\hline Jubilados & 58 & $(90,62)$ \\
\hline Baja & 2 & $(3,12)$ \\
\hline Paro & 2 & $(3,12)$ \\
\hline \multicolumn{3}{|l|}{ Escolaridad, n (\%) } \\
\hline Analfabeto & 7 & $(10,94)$ \\
\hline 5 años o menos & 22 & $(34,37)$ \\
\hline 6 años o más & 35 & $(54,68)$ \\
\hline \multicolumn{3}{|l|}{ Fase enfermedad, $\mathbf{n}(\%)$} \\
\hline - Leve & 19 & $(29,69)$ \\
\hline - Moderada & 20 & $(31,25)$ \\
\hline - Grave & 25 & $(39,06)$ \\
\hline \multicolumn{3}{|c|}{ Fase enfermedad-media edad (DE) } \\
\hline - Leve & 70,84 & $4 \quad(5,23)$ \\
\hline - Moderada & 73,75 & $5(4,53)$ \\
\hline - Grave & 80,52 & $2(4,44)$ \\
\hline Programas de ejercicio previos & 0 & $(0)$ \\
\hline
\end{tabular}

Tabla 2. Efectos del programa sobre las variables de capacidad física, funcional, capacidad cognitiva y CdV

\begin{tabular}{|c|c|c|c|c|}
\hline $\begin{array}{c}\text { Variables } \\
\text { Test n (64) } \\
\text { Rango }\end{array}$ & $\begin{array}{c}\text { Física y } \\
\text { Chair Stand Test } \\
{[0-]}\end{array}$ & $\begin{array}{l}\text { cional } \\
\text { Escala Barthel } \\
{[0-100]}\end{array}$ & $\begin{array}{c}\text { Cognitiva } \\
\text { MMSE } \\
{[0-30]}\end{array}$ & $\begin{array}{c}\text { Calidad de vida } \\
\text { SF-12 } \\
{[0-33]}\end{array}$ \\
\hline Pretest & $\begin{array}{l}7,21 \\
5,07\end{array}$ & $\begin{array}{l}72,65 \\
15,24\end{array}$ & $\begin{array}{c}14,65 \\
5,68\end{array}$ & $\begin{array}{c}22,68 \\
5,92\end{array}$ \\
\hline Postest & $\begin{array}{l}8,14 \\
6,25\end{array}$ & $\begin{array}{l}72,73 \\
16,49\end{array}$ & $\begin{array}{c}13,59 \\
6,60\end{array}$ & $\begin{array}{c}25,25 \\
8,50\end{array}$ \\
\hline$p^{*}$ & ,012 & ,901 & $\leq, 001$ & ,001 \\
\hline
\end{tabular}

MMSE: Mini Mental State Examination. 
Tabla 3. Efectos del programa y fase de la enfermedad: leve, moderada y grave

\begin{tabular}{|c|c|c|c|c|c|c|c|c|c|c|c|c|}
\hline \multirow{2}{*}{$\begin{array}{l}\text { Estadio (n) } \\
\text { Test }\end{array}$} & \multicolumn{4}{|c|}{ Leve (19) } & \multicolumn{4}{|c|}{ Moderada (20) } & \multicolumn{4}{|c|}{ Grave (25) } \\
\hline & $\begin{array}{l}\text { Chair } \\
\text { Stand } \\
\text { Test }\end{array}$ & $\begin{array}{l}\text { Escala } \\
\text { Barthel }\end{array}$ & MMSE & SF-12 & $\begin{array}{l}\text { Chair } \\
\text { Stand } \\
\text { Test }\end{array}$ & $\begin{array}{l}\text { Escala } \\
\text { Barthel }\end{array}$ & MMSE & SF-12 & $\begin{array}{l}\text { Chair } \\
\text { Stand } \\
\text { Test }\end{array}$ & $\begin{array}{l}\text { Escala } \\
\text { Barthel }\end{array}$ & MMSE & SF-12 \\
\hline \multirow[t]{2}{*}{ Pretest } & 11,42 & 83,42 & 21,57 & 27,15 & 9,1 & 80,50 & 15,75 & 22,80 & 2,52 & 58,20 & 8,52 & 19,20 \\
\hline & 3,32 & 8,82 & ,76 & 5,06 & 3,71 & 9,16 & 1,65 & 5,34 & 2,91 & 11,16 & 2,04 & 4,67 \\
\hline \multirow[t]{2}{*}{ Postest } & 14,52 & 87,36 & 21,47 & 34,63 & 9,4 & 79,50 & 14,95 & 25,30 & 2,24 & 56,20 & 6,52 & 18,08 \\
\hline & 3,15 & 7,14 & 1,39 & 3,11 & 4,61 & 8,72 & 2,32 & 6,24 & 2,89 & 11,11 & 2,45 & 5,34 \\
\hline$p^{*}$ & $\leq, 001$ &, 004 & ,772 & $\leq, 001$ & ,664 & 297 &, 022 &, 008 &, 454 & ,015 & $\leq, 001$ & ,280 \\
\hline
\end{tabular}

MMSE: Mini Mental State Examination.

el test de Barthel se observó una mejora significativa en la fase leve de la enfermedad $(p=0,004)$, mientras que, en las fases moderada y grave se observó una disminución no significativa en el primer caso $(p=0,297)$ y significativa en el segundo $(\mathrm{p}=0,015)$.

\section{Discusión}

En virtud de los resultados obtenidos se observa una mejora de la capacidad física y de la $\mathrm{CdV}$ del enfermo. Este efecto permite mantener las actividades básicas de la vida diaria en la fase leve y moderada de la enfermedad ${ }^{30,31}$. Este resultado es destacable considerando el impacto de la enfermedad sobre estas variables y el riesgo de dependencia ${ }^{12,32}$.

Se observa una relación inversamente proporcional entre la fase de la enfermedad y los efectos de la guía en todas las variables evaluadas. La capacidad cognitiva disminuye de forma continua y significativa en todas las fases evaluadas.

Analizando los resultados por fases, observamos que la variable de capacidad física y la CdV, mejoran tanto en la fase leve y en la fase moderada de la enfermedad, la capacidad funcional mejora en la fase leve y se mantiene estable en la fase moderada. Mientras que en la fase grave no se identifica ninguna mejora de las variables observadas. Deberían realizarse más estudios en esta línea para confirmar la tendencia de los resultados observados en esta investigación.

Aunque otros estudios confirman que a partir del programa de ejercicio mejora de la capacidad cognitiva de la persona enferma de Alzheimer ${ }^{33,34}$, los resultados de este estudio no han podido demostrarlo ${ }^{25,35}$. Este hecho se podría explicar por la avanzada edad de los enfermos, la diversidad de síntomas y la imposibilidad de trabajar en grupo. Además de los efectos identificados en la capacidad física y funcional ${ }^{36}$ destacamos la mejora en la $\mathrm{CdV}^{37}$. Este resultado es determinante y novedoso, y podría ser debido a la expresión vivencial e intencional de las capacidades físicas, psicológicas, sociales y emocionales de la persona enferma de Alzheimer. Un factor paradójico observado es la mejora de la CdV a pesar del impacto de los síntomas con los que conviven las personas enfermas. Esta situación se atribuye al propio efecto de la anosognosia ${ }^{38}$.

Consideramos que la pérdida de la autonomía no está causada únicamente por la enfermedad sino por la bradicinesia y el sedentarismo ${ }^{39}$. La mejora física y funcional transfiere en la autonomía de las ABVD y en los patrones motores básicos del enfermo ${ }^{40}$. Esta mejora, disminuye el riesgo de la triada, caída-fractura-dependencia ${ }^{41,42}$.

La GREPS se adapta de forma eficaz a las necesidades de la persona enferma en las fases iniciales de la enfermedad, debe optimizarse su eficacia en la fase grave de la enfermedad. Estos resultados justifican la promoción y adherencia a la guía ${ }^{43}$.

\section{Referencias}

1. Alzheimer's Disease International. World Alzheimer Report 2009. London: Alzheimer's Disease International, 2009.

2. Wimo A, Jonsson L, Gustavsson A, McDaid D, Ersek $\mathrm{K}$, Georges J, et al. The economic impact of dementia 
in Europe in 2008-cost estimates from the Eurocode project. Int J Geriatr Psychiatry 2011; 26 (8): 825-32.

3. López-Pousa S, Vilalta-Franch J, Garre-Olmo J, Pons S, Cucurella MG. [Characterisation and prevalence of the psychological and behavioural symptoms in patients with dementia]. Rev Neurol 2007; 45 (11): 683-8.

4. Burge E, Kuhne N, Berchtold A, Maupetit C, von GA. Impact of physical activity on activity of daily living in moderate to severe dementia: a critical review. Eur Rev Aging Phys Act 2012; 9 (1): 27-39.

5. Ferrara M, Langiano E, DiBrango T, DeVito E, DiCioccio L, Bauco C. Prevalence of stress, anxiety and depression in with Alzheimer caregivers. Health Qual Life Outcomes 2008; 6: 93.

6. Inouye K, Pedrazzani ES, Pavarini SC. [Alzheimer's disease influence on the perception of quality of life from the elderly people]. Rev Esc Enferm USP 2010; 44 (4): 1093-9.

7. Inouye K, Pedrazzani ES, Pavarini SC, Toyoda CY. Quality of life of elderly with Alzheimer's disease: a comparative study between the patients and the caregivers report. Rev Lat Am Enfermagem 2010; 18 (1): 26-32.

8. Olazaran J, Reisberg B, Clare L, Cruz I, Pena-Casanova J, Del ST, et al. Nonpharmacological therapies in Alzheimer's disease: a systematic review of efficacy. Dement Geriatr Cogn Disord 2010; 30 (2): 161-78.

9. Clare L, Linden DE, Woods RT, Whitaker R, Evans SJ, Parkinson $\mathrm{CH}$, et al. Goal-oriented cognitive rehabilitation for people with early-stage Alzheimer disease: a single-blind randomized controlled trial of clinical efficacy. Am J Geriatr Psychiatry 2010; 18 (10): 928-39.

10. Takeda M. Integration of drugs and non-pharmacological intervention to Alzheimer patients. Psychogeriatrics 2012; 12 (1): 1-2.

11. Van der RP. Complementary therapies in health care. Nurs Health Sci 2011; 13 (1): 4-8.

12. Burge E, Kuhne N, Berchtold A, Maupetit C, von GA. Impact of physical activity on activity of daily living in moderate to severe dementia: a critical review. Eur Rev Aging Phys Act 2012; 9 (1): 27-39.

13. Garber CE, Blissmer B, Deschenes MR, Franklin BA, Lamonte MJ, Lee IM, et al. Quantity and quality of exercise for developing and maintaining cardiorespiratory, musculoskeletal, and neuromotor fitness in apparently healthy adults: guidance for prescribing exercise. Med Sci Sports Exerc 2011; 43 (7): 1334-59.

14. Riebe D, Blissmer BJ, Greaney ML, Garber CE, Lees FD, Clark PG. The relationship between obesity, physical activity, and physical function in older adults. J Aging Health 2009; 21 (8): 1159-78.

15. Astell AJ, Clark SA, Hartley NT. Predictors of discharge destination for 234 patients admitted to a combined geriatric medicine/old age psychiatry unit. Int J Geriatr Psychiatry 2008; 23 (9): 903-8.

16. García-Mesa Y, López-Ramos JC, Giménez-Llort L, Revilla S, Guerra R, Gruart A, et al. Physical Exercise Protects Against Alzheimer's Disease in 3xTg-AD Mice. J Alzheimers Dis 2011; 24 (3): 421-54.

17. Penrose F. Can exercise affect cognitive functioning in Alzheimer's Disease? A review of the literature. Activities, Adaptation \& Aging 2005; 29 (4): 15-40.

18. Rey A, Canales I, Táboas M, Cancela JM. Consecuencias cognitivas del programa "memoria en movimiento". European Journal of Human Movement 2011; 22: 113-31.

19. Yu F, Kolanowski A. Facilitating aerobic exercise training in older adults with Alzheimer's disease. Geriatr Nurs 2009; 30 (4): 250-9.

20. Folstein MF, Folstein SE, McHugh PR. "Mini-mental state". A practical method for grading the cognitive state of patients for the clinician. J Psychiatr Res 1975; 12 (3): 189-98.

21. Huger D, Zieschang T, Schwenk M, Oster P, Becker C, Hauer K. Designing studies on the effectiveness of physical training in patients with cognitive impairment. Z Gerontol Geriatr 2009; 42 (1): 11-9.

22. Nelson ME, Rejeski WJ, Blair SN, Duncan PW, Judge JO, King AC, et al. Physical activity and public health in older adults: recommendation from the American College of Sports Medicine and the American Heart Association. Med Sci Sports Exerc 2007; 39 (8): 1435-45.

23. Tárraga L, Boada M. Volver a empezar. Ejercicios de estimulación cognitiva para enfermos de Alzheimer. 4-143. 1999. Glosa Ediciones. Ref Type: Serial (Book, Monograph).

24. Instituto de Mayores y Servicios Sociales. Reeducación psicomotriz y alzheimer. Ministerio de Sanidad, Política Social e Igualdad. Gobierno de España. 2011.

25. Rolland Y, Pillard F, Klapouszczak A, Reynish E, Thomas D, Andrieu S, et al. Exercise program for nursing home residents with Alzheimer's disease: a 1-year randomized, controlled trial. J Am Geriatr Soc 2007; 55 (2): 158-65.

26. Blesa R, Pujol M, Aguilar M, Santacruz P, Bertrán-Serra I, Hernández G, et al. Clinical validity of the 'minimental state' for Spanish speaking communities. Neuropsychologia 2001; 39 (11): 1150-7.

27. Jones CJ, Rikli RE, Beam WC. A 30-s chair-stand test as a measure of lower body strength in community-residing older adults. Res Q Exerc Sport 1999 Jun; 70 (2): 113-9.

28. Mahoney FJ, Barthel DW. Functional evaluation: The Barthel Index. Md State Med J 1965; 14: 61-5.

29. Vilagut G, Valderas JM, Ferrer M, Garín O, López-García E, Alonso J. [Interpretation of SF-36 and SF-12 ques- 
tionnaires in Spain: physical and mental components]. Med Clin (Barc) 2008; 130 (19): 726-35.

30. Steinberg M, Leoutsakos JM, Podewils LJ, Lyketsos CG. Evaluation of a home-based exercise program in the treatment of Alzheimer's disease: the Maximizing Independence in Dementia (MIND) study. Int J Geriatr Psychiatry 2009; 24 (7): 680-5.

31. Stevens J, Killeen M. A randomised controlled trial testing the impact of exercise on cognitive symptoms and disability of residents with dementia. Contemp Nurse 2006; 21 (1): 32-40.

32. Arkin SM. Student-led exercise sessions yield significant fitness gains for Alzheimer's patients. Am J Alzheimers Dis Other Demen 2003; 18 (3): 159-70.

33. Arkin S. Language-enriched exercise plus socialization slows cognitive decline in Alzheimer's disease. Am J Alzheimers Dis Other Demen 2007; 22 (1): 62-77.

34. Gallucci M, Limbucci N, Catalucci A, Caulo M. Neurodegenerative diseases. Radiol Clin North Am 2008; 46 (4): 799-817.

35. Taylor AH, Cable NT, Faulkner G, Hillsdon M, Narici M, Van Der Bij AK. Physical activity and older adults: a review of health benefits and the effectiveness of interventions. J Sports Sci 2004; 22 (8): 703-25.

36. Hauer K, Schwenk M, Zieschang T, Essig M, Becker C, Oster P. Physical training improves motor performance in people with dementia: a randomized controlled trial.
J Am Geriatr Soc 2012; 60 (1): 8-15.

37. Sorensen LV, Waldorff FB, Waldemar G. Social participation in home-living patients with mild Alzheimer's disease. Arch Gerontol Geriatr 2008; 47 (3): 291-301.

38. Vogel A, Mortensen EL, Hasselbalch SG, Andersen BB, Waldemar G. Patient versus informant reported quality of life in the earliest phases of Alzheimer's disease. Int J Geriatr Psychiatry 2006; 21 (12): 1132-8.

39. Littbrand H, Lundin-Olsson L, Gustafson Y, Rosendahl E. The effect of a high-intensity functional exercise program on activities of daily living: a randomized controlled trial in residential care facilities. J Am Geriatr Soc 2009; 57 (10): 1741-9.

40. Tinetti ME, Kumar C. The patient who falls: "It's always a trade-off". JAMA 2010; 303 (3): 258-66.

41. Logsdon RG. Dementia: psychosocial interventions for family caregivers. Lancet 2008; 372 (9634): 182-3.

42. McGough EL, Kelly VE, Logsdon RG, McCurry SM, Cochrane BB, Engel JM, et al. Associations Between Physical Performance and Executive Function in Older Adults With Mild Cognitive Impairment: Gait Speed and the Timed "Up \& Go" Test. Phys Ther 2011 May 26.

43. Paterson DH, Warburton DE. Physical activity and functional limitations in older adults: a systematic review related to Canada's Physical Activity Guidelines. Int J Behav Nutr Phys Act 2010; 7: 38. 